\title{
Reversal of Cell Death in the Laboratory: Reversal of Clinical Death in the Emergency Room
}

\section{Benjamin J. Scherlag*, Ronald A. Scherlag, Khaled Elkholey, Tarun Dasari}

Department of Medicine, University of Oklahoma Health Sciences Center, Oklahoma, United States. *Corresponding Author: Benjamin J. Scherlag, 1200 Everett Drive (6E103), Oklahoma City, OK, USA.

\section{Abstract}

Background: A previously described dwarfform of the unicellular organism, Stentor coeruleus has been shown to undergo apoptosis, cell death, when placed in a toxic solution. Within minutes these cells progressively released a replicate image.

Methods: Deep well slides $(n=10)$ were filled with Stentor containing culture media and allowed to evaporate for 24 hours. Each slide was rehydrated with commercially obtained sterile Stentor media. Within 24 hours each slide was inhabited by mobile dwarf forms. The liquid contents from each of 5 slides was transferred into a small beaker and an aliquot of chlorinate tap water. After mixing, the contents were distributed to 5 deep well slides and sealed with a snap cover, active group. The liquid contents from the other 5 slides without the tap water served as the control group.

Results: The cells of the active group lost mobility and became stationary within 8-15 minutes. The stationary cells progressively released a replicate image and when fully formed plate magnets applied across the slides induce a magnetic field (ranging from 60-90 milliGauss) allowing retraction of the replicate back into the apoptotic cell. Droplets from these deep well slides placed in a favorable solution was followed by restoration of the living, mobile state.

Conclusions: In the present study we were able to induce, via a magnetic field, retraction of the fully developed replicate back into the dead cell with the subsequent restoration of the living state in a favorable environment. This sequence of events in living cells is analogous to the Out-of-Body phenomenon described in patients resuscitated from clinical death. The medical implications of these finding are discussed.

Keywords: Stentor coeruleus, Dwarfforms, Cell death, Replicate image, Restoration of the living state

Introduction: We subjected unicellular dwarf forms of the organism, Stentor coeruleus, to a toxic environment in order to produce evidence of cell death, apoptosis. In previous studies, cell death initiated a progressive release of a replicate image of the cell. In this report we induced a retraction of the replicate image back into the dead cell. Methods: Deep well slides $(n=10)$ were filled with Stentor containing culture media and allowed to evaporate for 24 hours. Rehydration was achieved by refilling each slide with commercially obtained sterile Stentor media. Within 24 hours each slide was inhabited by mobile dwarf forms. The liquid contents from each of 5 slides was transferred into a small beaker with an aliquot of chlorinate tap water. After mixing, the contents were distributed to 5 deep well slides and sealed with a snap cover, active group. The liquid contents from the other 5 slides without the tap water served as the control group.

Results: The cells of the active group lost mobility and became stationary within 8-15 minutes. The stationary cells progressively released a replicate image and when fully formed plate magnets applied across the slides induce a magnetic field (60-90 milliGauss) allowing retraction of the replicate back into the apoptotic cell. Droplets from these deep well slides placed in a favorable solution was followed by restoration of the living, mobile state.

Conclusions: In the present study we were able to induce, via a magnetic field, retraction of the fully developed replicate back into the dead cell with the subsequent restoration of the living state when these cells were introduced to a favorable environment. This sequence of events in living cells is analogous to the Out-of-Body phenomenon described in patients resuscitated from clinical death. The medical implications of these finding are discussed. 
Reversal of Cell Death in the Laboratory: Reversal of Clinical Death in the Emergency Room

\section{INTRODUCTION}

The metaphysical literature is replete with subjective narratives of an Out-of-Body experience, particularly from individuals who have been resuscitated from a terminal event. The classic story consists of a replicate of the individual's body and consciousness separating from their physical form, remaining for some period of time in the vicinity and returning to their physical body upon successful resuscitation. In a previous report, we found that a dwarf form of the unicellular animal, Stentor coeruleus under specific conditions would release micronuclei which developed into mobile dwarf forms [1]. We recently extended these findings by demonstrating that these dwarf forms undergo apoptosis when placed in a toxic solution similar to the experiments performed by Sun et al. as a preliminary stage for inducing "anastasis" i.e., reversal of cell death [2]. In our studies, the moribund cells, within 8-15 minutes, progressively released a replicate image which faded away after 20-30 minutes [3]. In the present study, we were able to induce retraction of the fully developed replicate back into the dead cell with the subsequent restoration of the living state.

\section{METHODS}

Stentor cultures were obtained from the Carolina Biological Supply Company, Burlington NC, USA. Dwarf organisms were obtained as described in a previous report [1]. Briefly, deep well slides ( $n=9)$ were filled with Stentor containing culture media and allowed to evaporate for 24 hours. Rehydration was achieved by refilling each slide with sterile commercially obtained Stentor media*, to the active group. A plastic spacer, 2.5 $\mathrm{mm}$ width, was placed over each uncovered slide and a glass plate over all the slides prevented evaporation of the unsealed contents (Figure1). The next day, each of the deep well slides was examined microscopically and found to contain many mobile dwarf cells as well as progenitor cells each of which gave rise to 4 dwarfs [3].

A pipette was used to transfer the liquid contents from each of 5 slides into a small beaker. An aliquot of tap water* was added to the beaker and the contents thoroughly mixed. Each of 5 deep well slides was filled with the mixture and enclosed with a snap cover, active group. The liquid contents from the other 5 slides without the tap water served as the control group. The slides from each group were viewed by a Nikon TMR, inverted microscope and the other placed on the stage of a OMAX video microscope.

Micro-photographs and videos were recorded by a camera attachment to the Nikon microscope and from the OMAX microscope, respectively.

*City of Oklahoma City. 2017 Drinking Water Quality Report. Average chlorine levels, $3.32 \mathrm{ppm}$ used to disinfect the city water.

\section{RESULTS}

Over a period of 8-15 minutes the cells of the active group lost mobility and their inclusions also cease any movement. The stationary cells show sequential changes consisting of the progressive development of a replicate image being released from the original cell Figure 1. Within 20-30 minutes the replicate gradually fades. A detailed description of these changes with time is provided in [3]. None of the control group showed these changes. When most of the cells in the active group reached full replicate development (8-15 minutes) a plate magnet, 3975 Gauss, was placed on either side of the deep well slide in order to create a magnetic field (+/-) across the slide. Using a hand held magnetometer we registered the field strength ranging from 60-90 milligauss.

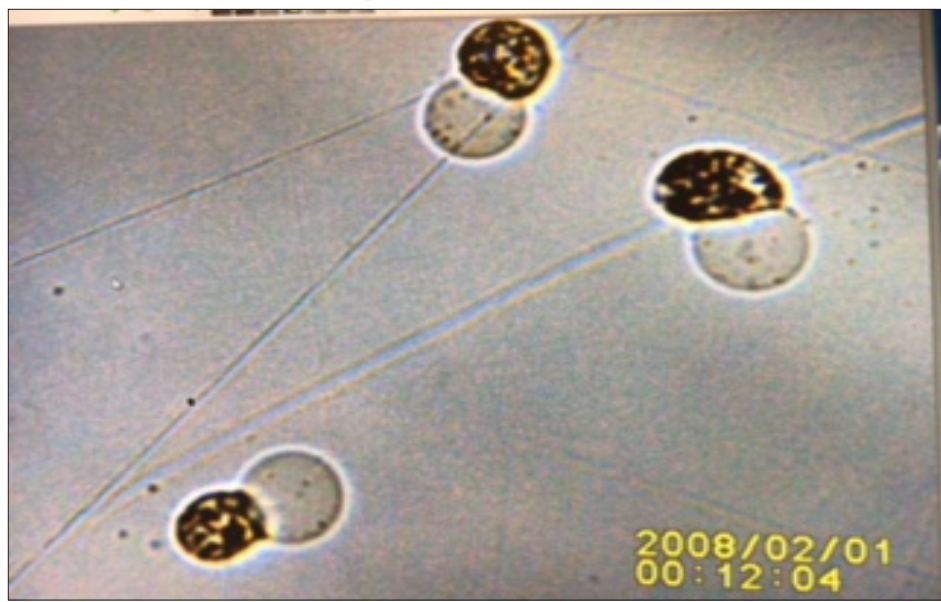

Fig1. Image showing a collection of moribund dwarf cells in various stages of replicate formation. The cell diameters ranged from 25 to 40 microns. 
Reversal of Cell Death in the Laboratory: Reversal of Clinical Death in the Emergency Room

When the replicate image was at its peak development we deployed 2 plate magnets, each 3795 gauss on either side of the deep well slide to create a magnetic

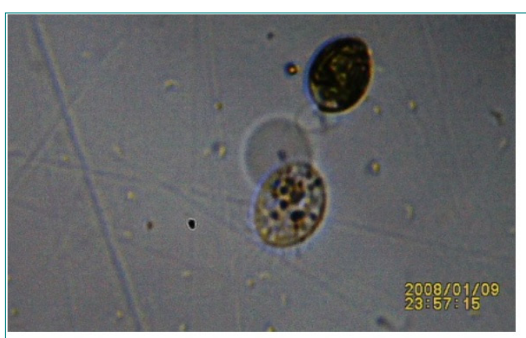

A

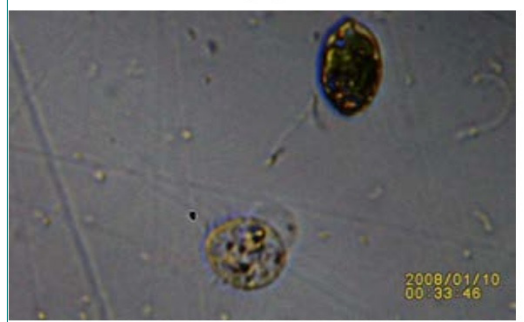

D

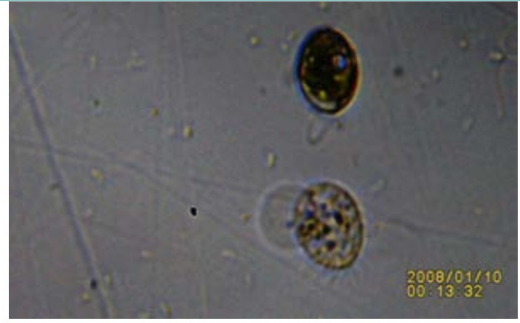

B

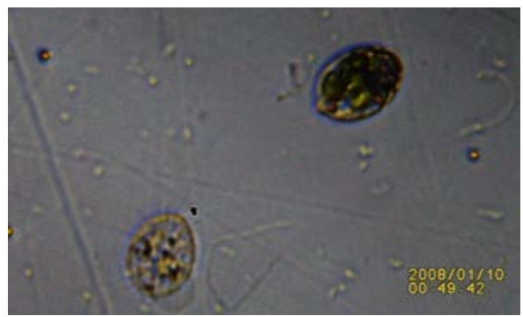

E field. Figure 2 shows a time lapse over a 20 minute period during which the replicate was progressively retracted back into the "dead" cell.

Fig2. Time lapse illustration showing the replicate beginning to move back into the "dead" cell. Note the rotation of the cell as the replicate retracts $(A-F)$.

A small droplet of the solution containing these retracted cells was then placed into eight separate deep well slides each of which was filled with a favorable medium devoid of cells and snap covered. Twenty four- 48 hours later the slides were microscopically examined.

https://www.dropbox.com/s/mubep89ds9m4krz/ Media1.mp4?dl=0.

Fig3. (video) shows the restoration of the dwarf cell into their previous mobile states.

Of the eight deep well slides which contained the recombined cells $7 / 8$ showed restoration of the active mobile states.

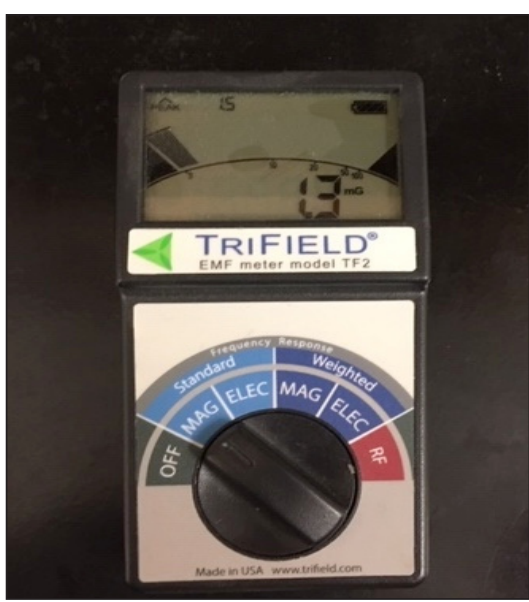

A

\section{Discussion}

\section{Major Findings}

The results of our present findings provide an extension of our previous studies showing that when the dwarf Stentor cells were subjected to a toxic solution, the dying/dead cell released a replicate body which in a relatively short time faded from view. At the optimum level of replicate formation, the application of a magnetic field, in these studies $\sim 73$ milligauss, resulted in a gradual retraction of the replicate back into the "dead" cell (see Figure 3A, B).

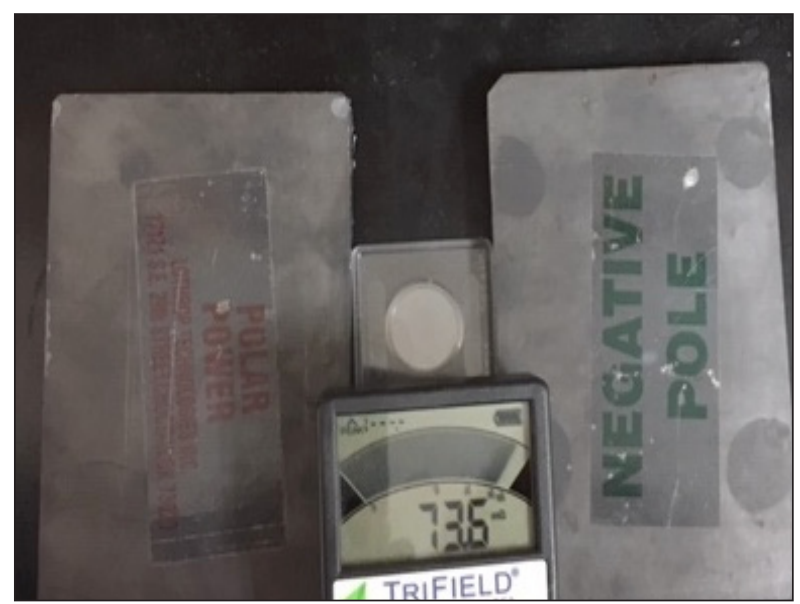

$\mathrm{B}$

Fig3. A. Illustration of the TriField magnetometer. B. Two plate magnets placed on either side of the deep well slide containing the dwarf Stentor cells. See text for discussion. 
Small droplets of the solution from these deep well slides were then applied to a clean slide each of which $(n=8)$ was filled with a solution favorable for the dwarfs but devoid of cells. Within the next day, mobile dwarf cells were observed during microscopic examination.

\section{Background}

In an initial report we documented that dwarf cells (25-40 microns) of the unicellular organism, Stentor coeruleus, could be cultured from the evaporated forms of the large Stentor cells (750-1000 microns) particularly those showing disrupted membranes [1]. The release of micronuclei from these cells were shown to form progenitor cells in a favorable solution in which the progenitor cells, in a unique process, gave rise to 4 actively, mobile dwarf cells. In subsequent studies we found that these dwarf cells when subjected to a toxic solution, i.e., chlorinated tap water, would undergo apoptosis, programmed cell death including the development of transient blebbing [4]. Of greater interest, the dying/dead cell released a progressively developing replicate within 8-15 minutes that faded after 20-30 minutes [3].

\section{The Present Study}

We hypothesized that by reversing the replicate formation and having it retract back into the apoptotic cell, the living state of these organisms could be restored. This unique form of cellular restoration, called anastasis (Greek for 'rising to life') has been documented by others [5]. Sun G and Montell DJ [2] described anastasis as a "process by which cells survive ...following transient exposure to a lethal dose of an apoptotic stimulus."

It should be noted that in previous studies replicate images have been documented in plant and animal tissues [6] and in unicellular plants [7]. We have not reproduced but rather simulated the out-of-body phenomenon that has been described at the life/ death junction across the evolutionary spectrum. This response has been documented in cultured cancer cell lines among others. The discovery of the reversal of cell death in unicellular organisms and in mammalian tissues "suggests that it is an ancient and evolutionarily conserved cellular process." [2]

Finally, the formation of a replicate from a moribund physical body at the time of death, is analogous to the basic duality inherent in Quantum theory. Indeed, a recent theoretic presentation stated that, "Quantum biology is emerging as a new field at the intersection between fundamental physics and biology" [8].

\section{Clinical Implications}

The sequence of events in our previous study [3] taken together with the results of the present report represent the complete cycle of the Out-ofBody experience detailed in the many paranormal descriptions [9-11]. The present study in a unicellular organism closely resembles the descriptions of the Out-of-body experiences in patient undergoing resuscitation in emergency rooms. Could the observations made in these studies serve as feasible method to increase the number of patients successfully resuscitated in emergency rooms? One approach might be the placement of a device such as a Helmholtz coil (Figure 4, Magneceutical Health, Clearwater, Fla) to enclose the patient's head so as not to interfere with the resuscitation procedure. Using milligauss levels of magnetic fields the patient's out of body replicate might be forced back into the physical body, thus accomplishing a successful resuscitation.

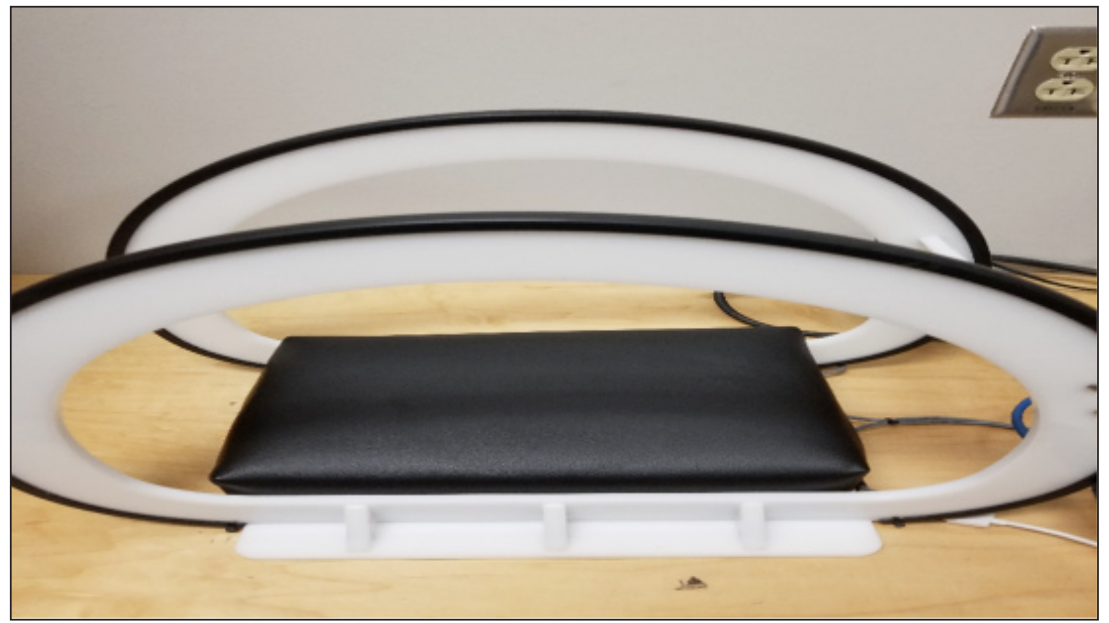

Fig4. Illustration of a Helmholtz coil for delivery of low-level electromagnetic fields to the head and neck of the patient in the emergency room undergoing resuscitation procedure after clinical death. 
Reversal of Cell Death in the Laboratory: Reversal of Clinical Death in the Emergency Room

\section{LIMITATIONS}

Questions remain as to the actual death of the dwarf subjected to a toxic solution such as chlorinated water. In their studies Sun and Montell [2] designated the dead cells as having undergone apoptosis and had reached the point of near death since the enzyme, which they identified as "executioner caspase" had not been activated. We have not, as yet, determined the role of this enzyme in our apoptotic cells.

\section{ConCLUSIONS}

We previously reported that a dwarf form of the unicellular animal, Stentor coeruleus, when placed in a toxic environment causing cell apoptosis, i.e, cell death, would release a replicate of the cell. In this follow-up study we were able to induce retraction of the fully developed replicate back into the dead cell with the subsequent restoration of the living state when these cells were introduced to a favorable environment. This sequence of events in living cells is analogous to the Out-of-Body phenomenon described in patients resuscitated from clinical death.

\section{REFERENCES}

[1] Scherlag BJ. The Life Cycle of Dwarf Stentors. Letters in Health and Biological Sciences. 2017;2: 1-4.

[2] Sun G,Montell DJ.Q\&A:Cellularnear death experienceswhat is anastasis? BMC Biol. 2017; 15: 92-97

[3] Scherlag BJ. Scherlag RA, Elkholey K, Jacobson JI. Replicate Separation from Dead Dwarf Cells of Stentor coeruleus. Biosystems 2019;181: 27-30.
[4] Charra GT. A short history of blebbing. J Microsc. 2008;231: 466-478

[5] Tang HL, Tang HM, Mak KH, Hu S, Wang SS, Wong KM, et al. Cell survival, DNA damage, and oncogenic transformation after a transient and reversible apoptotic response. Mol Biol Cell. 2012;23: 2240-2252

[6] Scherlag BJ, Elkholey K, Embi AA, Jacobson JI, Scherlag JB, Sahoo K, Po SS. Electromagnetic Energy Emanating from Plant and Animal Tissues in the form of replicate images

[7] Scherlag BJ, Sahoo K, Embi, AA. Replicate Imaging of a Unicellular Plant through a Glass Barrier Using Fine Iron Particles: Evidence for Electromagnetic Energy Transfer. Letters in Health and Biological Sciences 2017;10: 82-85

[8] Bordonaro M, Ogryzko v. Quantum biology at the cellular level-Elements of the research program. Biosystems. 2013;112: 13-30.

[9] Moody RA. Life After Life. 1975. Mockingbird Books

[10] Ring K. Heading Towards Omega: In Search of the Meaning of the Near-Death Experience. 1985. HarperCollins

[11] Parnia S, Spearpoint, K; de Vos, G; Fenwick, P; et al. (2014). "AWARE-AWAreness during RE suscitation-a prospective study". Resuscitation. 2014; 85 : 1799-805.

Citation: Benjamin J. Scherlag, Ronald A. Scherlag, Khaled Elkholey, Tarun Dasari. Reversal of Cell Death in the Laboratory: Reversal of Clinical Death in the Emergency Room. Archives of Emergency Medicine and Intensive Care. 2020; 3(1): 21-25.

Copyright: (c) 2020 Benjamin J. Scherlag, Ronald A. Scherlag, Khaled Elkholey, Tarun Dasari. This is an open access article distributed under the Creative Commons Attribution License, which permits unrestricted use, distribution, and reproduction in any medium, provided the original work is properly cited. 